Published in final edited form as:

Crit Care Med. 2018 September ; 46(9): e963-e964. doi:10.1097/CCM.0000000000003243.

\title{
Response to Oud
}

Patrick D. Tyler, MD ${ }^{1}$, Barret Rush, MD, MPH², and Leo Anthony Celi, MD, MS, MPH ${ }^{3,4}$

${ }^{1}$ Department of Emergency Medicine, Beth Israel Deaconess Medical Center, Boston, MA

2Department of Medicine, University of British Columbia, Vancouver, BC, Canada

${ }^{3}$ Division of Pulmonary, Critical Care, and Sleep Medicine, Department of Medicine, Beth Israel

Deaconess Medical Center, Boston, MA

${ }^{4}$ Institute for Medical Engineering and Science, Massachusetts Institute of Technology,

Cambridge, MA

\section{Keywords}

sepsis; inter-hospital transfer; health systems research; health care disparities

\section{To the editor}

We thank Dr. Oud for his comments (1) on our article (2).

To the first point, we identified patients with sepsis using the Angus methodology, and not through the use of explicit International Classification of Diseases (ICD) coding for sepsis. There is no perfect way to retrospectively identify patients with sepsis, as we addressed in another paper (3). The Angus methodology was recently validated by our group albeit on a different database in another paper (3). Although it is imperfect, it is among the most widely used and accepted strategies to identify patients with sepsis in administrative databases. We acknowledged this limitation in our paper: "because of the sensitivity of the ICD-9 administrative code for sepsis, some patients with sepsis may have been inadvertently excluded; however, the ethnicity of patients not captured by this administrative approach should be random, and thus should not affect our findings on disparity" (2).

Regarding the second point about the fidelity of the do-not-resuscitate (DNR) status during the study period, we analyzed three years of data with this variable available (2010, 2011, and 2012), not two, representing roughly half of the studied population. Furthermore, the odds ratio for DNR status was $0.15-0.25$ in the first model, and 0.16-0.26 in the second model. This finding (with a confidence interval far from 1) is consistent with what we expect a priori: that patients who prefer some care limitations, and their providers, are less likely to pursue inter-hospital transfer (IHT). As with our other results, we present this finding as preliminary, and agree that it requires further investigation.

Corresponding author: Patrick D. Tyler, pdtyler@bidmc.harvard.edu.

Copyright form disclosure: Drs. Tyler and Celi received support for article research from the National Institutes of Health. Dr. Rush has disclosed that he does not have any potential conflicts of interest. 
Regarding the third point about adjusting for severity of illness (SOI), we explicitly stated that as a limitation; unfortunately, the information is not available in the Nationwide Inpatient Sample. Minority patients with sepsis are, on average, sicker; the purpose of transfer is to move the sickest patients to larger centers with more experience. So if minority patients are sicker on average, and the purpose of transfer is to move sicker patients to experienced centers, we should see higher rates of transfer in minority patients. Yet we found that minorities were less likely to be transferred. The absence of SOI scoring should not blunt our interest in this question; rather, we need to dig deeper, and if the disparity finding is confirmed, we need to investigate the causes and make changes where appropriate.

We do agree with Oud on his final point: that further study is needed. Our goal was not to provide a definitive answer as to whether racial disparities exist in IHT. We hope our paper brings the issue to the forefront and piques the interest of the critical care community. We welcome the future contributions of Dr. Oud and others to replicate, refute or build upon our findings.

\section{References}

1. Oud L. Disparities in the Propensity for Interhospital Transfer among Mechanically Ventilated Septic Patients. Crit Care Med. 2018 in press.

2. Tyler PD, Stone DJ, Geisler BP, McLennan S, Celi LA, Rush B. Racial and Geographic Disparities in Interhospital ICU Transfers*. Crit Care Med [Internet]. 2018 Jan; 46(1):e76-80. [cited 2018 Apr 20]. Available from: http://www.ncbi.nlm.nih.gov/pubmed/29068859.

3. Johnson AEW, Aboab J, Raffa JD, Pollard TJ, Deliberato RO, Celi LA, et al. A Comparative Analysis of Sepsis Identification Methods in an Electronic Database. Crit Care Med [Internet]. 2018 Apr; 46(4):494-9. [cited 2018 Apr 20]. Available from: http://insights.ovid.com/crossref? an=00003246-201804000-00002. 